\title{
MATERIALS DATA BASES
}

are going to transform your lifestyle

A meeting was held at Fairfield Glade, Tenn., Nov. $5-11$ that will change the course of materials science in the next decade. The National Academy of Science and the National Bureau of Standards sponsored an international gathering to determine how to develop an international system for computerized materials data bases. Your representative at the meeting was the 1982 president of the Materials Research Society, Clyde J.M. Northrup, Jr. One of the results of the meeting was the recommendation that a central "gateway" system be established in the United States and sponsored by the National Academy of Sciences, with compatible systems being organized in Japan and Europe. Management of technical information is already affecting industry where the productivity of materials scientists who daily use the computers has increased remarkably, while the total number of people being employed has been reduced.
Objectives of the U.S. "gateway" system will be to provide materials scientists with a single user-friendly source of information on materials. The gateway will tie existing and new data bases together, allowing users immediate access to a large amount of up-to-date information and requiring that only one protocol be learned. It is anticipated that information stored in the data bases will have multiple levels of critical evaluation, allowing users to discriminate between vendor claims, refereed measurements and certified standards. The individual data bases would be maintained by technically competent administrators and tied to the national system by a network. This will allow users to locate and retrieve information stored at locations around the world and then to "down load" needed data into their computers for data manipulation and analysis.

Users of information in a computer format are increasing rapidly. For example, data bases are used for surveying materials properties much as one would thumb through a reference manual, and also for initial selection of materials. Final materials selection based on optimization due to critical materials properties, availability, cost and so on may also be done using computer data bases. Materials information may be fed directly to a computer-aided-design (CAD) or computer-aided-manufacturing (CAM) facility.

Having complete, current information readily available allows materials scientists to identify gaps in knowledge. to suggest alternative materials, to reduce redundancy in measurements and to plan "smart" experiments - that is, to make the critical measurements using statistical techniques to identify sensitive parameters and to permit interpolation elsewhere. Materials scientists emerging today no longer use computers only to record and plot data:

[Continued on Page 2]

\section{MATERIALS DATA BASE INFORMATION}

Which reference manuals do you utilize most frequently?

What materials' properties are of most
importance to you?
Chemical
Electrical
Mechanical
Other (please specify)

What materials' properties are of secondary importance?

Chemical

Electrical

Mechanical

Other (please specify)

What formats are most helpful to you?

Tables

Graphs

On-line digitized data

Other (please specify)
What do you use the information for?

Theoretical calculations

\begin{tabular}{l}
\hline Systems modeling \\
\hline Materials selection \\
\hline Design specifications \\
\hline Other (please specify)
\end{tabular}

Do you have a computer data base that you find useful?

If yes, please identify

\section{Other comments}

(Optional)

Name

Address

\section{Telephone}

Return this questionnaire to:

Clyde J.M. Northrup, Jr.

Sandia National Laboratories

Albuquerque, NM 87185 


\section{H.J. LEAMY IS 1983 MRS PRESIDENT}

\section{Bell Laboratories materials scientist is the Society's new leader}

The Materials Research Society's chief executive officer for 1983 is Harry J. Leamy, supervisor of the projection display development group at Bell Laboratories in Murray Hill, New Jersey. An executive and a materials scientist of broad experience, Harry has long been active in the leadership of the MRS, for example co-chairing with J.M. Poate the first MRS symposium on laser-solid interactions, which was held in 1979.

Harry Leamy (pronounced LAY-me) received formal training as a metallurgist from the University of Missouri at Rolla (B.S. 63) and Iowa State University (Ph.D. 67). His thesis research into the mechanical behavior of long range ordered alloys was followed by post doctoral research on the same topic at the Max Planck Institut fuer Metallforschung in Stuttgart.

Harry accepted employment at Bell Laboratories as a member of the materials physics research department in 1969. His research activities included transmission and scanning electron microscopy, metallic glasses, thin film microstructures, permanent magnet materials, crystal growth and amorphous, ferrimagnetic materials.

He spent a sabbatical year at the Philips Research Laboratories in Eindhoven, and upon his return to Bell in 1976 became a member of the electronics materials research department. His research activities for the last five years have focused on silicon. He has studied defects in semiconductor silicon and their effects on device behavior. More recently, he has devoted himself to laser processing of silicon for device applications. He has authored more than a hundred technical articles and has been granted a dozen patents.

Harry has been a member of the MRS since the Society's inception. Having participated in MRS symposia, he was asked in 1979 to co-chair the symposium on laser-solid interactions. Two years later, he and Woody White co-chaired the annual meeting, and Harry also co-chaired, with Carl Seager and Gordon Pike, the symposium on grain boundaries in semiconductors. Subsequently Harry was elected MRS vice president for 1982.

As vice president, Harry was a member of the Publications Committee

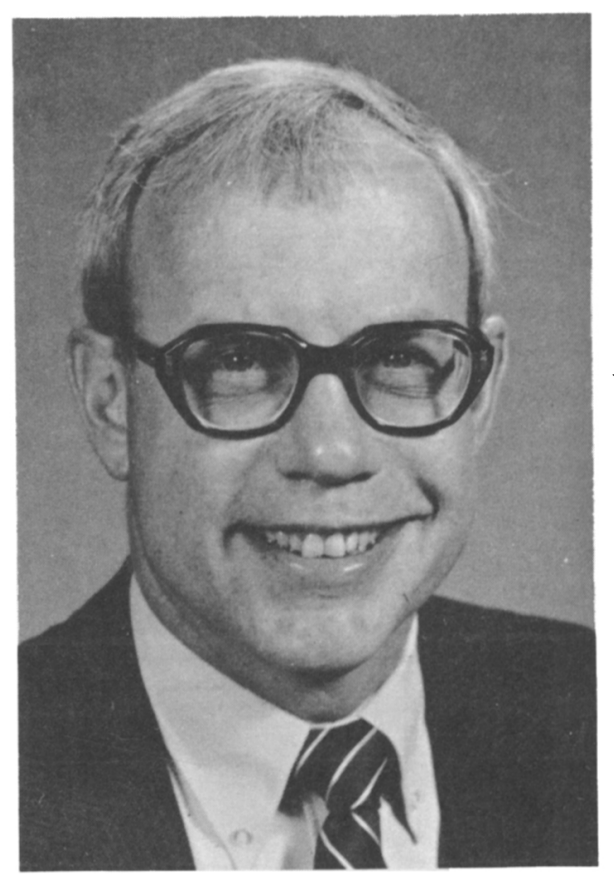

\section{H.J. LEAMY}

(which he had chaired in 1981), and was active in arranging the publication of MRS symposia as a Proceedings series. He likewise fostered the regularization of publication of this Bulletin, and plays a fair game of handball.

\section{MATERIALS}

\section{[Continued from Page 11]}

they use them to plan their experiments.

The changes occurring in materials science will, no doubt, create as well as eliminate some jobs, and a formidable educational task is before us. Training existing materials scientists will require both awareness programs to alert people to the change that is occurring, as well as educational programs to provide them with guidance in the new technology.

The current U.S. schedule is to have a pilot network between materials science data bases tested in 1983, a demonstration system at the national level (possibly linked to data bases abroad) within three years, and a national gateway system operational within five years. There are many computerized materials data bases extant but as the gateway will serve materials scientists for a long time it is essential that the network be planned carefully to permit users rapid and efficient access to the information they seek. Those who are planning the national system need your guidance in selecting the information that:

- Should be included in the initial data bases in the network;

- Would be heldful but of secondary importance among materials data bases; and

- Should be formatted to be of most value to you (i.e., graphs, tables, etc.).

If you have or know of materials data bases that might be useful in this regard please convey that information to us. The members of the Materials Research Society comprise a broad range of backgrounds. Your views are highly regarded. Please take a few minutes to fill out the questionnaire an return it to us.

WE NEED YOUR HELP! 